\title{
How could the Io footprint disappear?
}

\author{
S.L.G. Hess ${ }^{\mathrm{a}, *}$, B. Bonfond ${ }^{\mathrm{b}}$, P.A. Delamere ${ }^{\mathrm{c}, 1}$ \\ a LATMOS, Université Versailles-St Quentin, IPSL/CNRS, 11 Boulevard d'Alembert, 78280 Guyancourt, France \\ ${ }^{\mathrm{b}}$ LPAP, Université de Liège, Belgium \\ ${ }^{\mathrm{c}}$ University of Alaska, Fairbanks, USA
}

\section{A R T I C L E I N F O}

\section{Article history:}

Received 23 April 2013

Received in revised form

19 August 2013

Accepted 20 August 2013

Available online 29 August 2013

Keywords:

Jupiter-Io interaction

Io footprint

UV aurora

Dispersive Alfvén waves

\begin{abstract}
A B S T R A C T
The interaction of Io with the Jovian magnetosphere is the best known - and the most intense - case of satellite-magnetosphere interaction. The interaction involves a power of more than a TeraWatt, a few percent of which are transferred to electrons. These electrons precipitate in the Jovian ionosphere where they light-up bright auroral emissions (several 100's kR to a few 1000's kR). The brightness of the Iocontrolled UV auroras is known to vary, due to the Jovian magnetic field tilt, which induces longitudinal variations, and due to the Io torus ever changing parameters (possibly due to Io's volcanic activity), which induce a temporal variation. As Io-controlled UV auroras have been monitored for a long time, the variation of their brightness is well-documented, and the typical amplitude of these variations has been established. However, on June 7, 2007 an unusual event occurred in the plasma torus surrounding Io, which triggered its own UV emissions on Jupiter in a region mapping to Io's orbit. When Io reached that region, Io's auroral footprint disappeared, its brightness dimming by at least a factor of three to be below the background aurora brightness. Both the auroral event at such a low latitude and the Io footprint disappearance are events that have never been observed before and should be quite rare. However, the question of how the bright Io footprint becomes that weak remains.

From a theoretical point-of-view, the Io-Jupiter interaction has been widely studied. In the 80 's, it was shown that Alfvén waves are radiated from Io, carrying currents to Jupiter. In the late-2000's, studies showed that dispersive Alfvén waves were likely to cause the acceleration of the electrons powering the auroral emissions, although Io-scale Alfvén waves should be non-dispersive. More recently, a model was built which permits one to compute the ratio between dispersive and non-dispersive waves in the auroral region for satellite-magnetosphere interactions, and thus the brightness of the related aurorae. We use this model to investigate which variation of the interaction parameters could lead to the Io footprint disappearance.
\end{abstract}

(c) 2013 Elsevier Ltd. All rights reserved.

\section{Introduction}

Io is the innermost Galilean satellite of Jupiter, and is the most active volcanic body of our solar system. It releases about 1 ton/s of neutral matter in the Jovian magnetosphere, roughly half of which is ionized and remains frozen in the Jovian magnetic field (Bagenal, 1997; Saur et al., 2003; Thomas et al., 2004, and references therein) and forms a dense plasma torus. The plasma torus concentrates in the centrifugal equator and rotates with the period of Jupiter (9h55) (Schneider and Trauger, 1995), whereas Io orbits with a Keplerian period of $42.5 \mathrm{~h}$ in the jovigraphic equator, inclined by $\sim 7^{\circ}$ from the centrifugal equator.

\footnotetext{
* Corresponding author. Tel.: +33180285063.

E-mail address: sebastien.hess@latmos.ipsl.fr (S.L.G. Hess).

${ }^{1}$ Formerly at: LASP, University of Colorado, Boulder, USA.
}

The motion of Io relative to the Jovian magnetic field - with a velocity of $V_{\text {Io }}=57 \mathrm{~km} / \mathrm{s}$ - and the perturbation of the plasma flow generate an electric field $E_{\mathrm{Io}}=-V_{\mathrm{Io}} \times B$, inducing a current $J$ (Goldreich and Lynden-Bell, 1969) which closes in the Jovian ionosphere. This current leads to the acceleration of electrons which precipitate in the Jovian ionosphere and generate the auroral footprint of Io through collisions with neutrals. These intense auroral emissions are observed from Infrared (Connerney et al., 1993) to the UV domain (Prangé et al., 1996; Clarke et al., 1996). The UV observations have the best spatial and temporal resolutions (Bonfond et al., 2007, 2009; Bonfond, 2010).

The Io footprint, a consequence of the Io interaction with the Jovian magnetosphere, is one of the brightest features of the Jovian aurorae (Fig. 1). It has been observed numerous times in the UV and its typical brightness, and the typical amplitude of its variation are well-defined (Bonfond et al., in press, and references therein, Figure 1c). This footprint is clearly distinct from all the other features, and in particular from the main auroral oval, so that the 

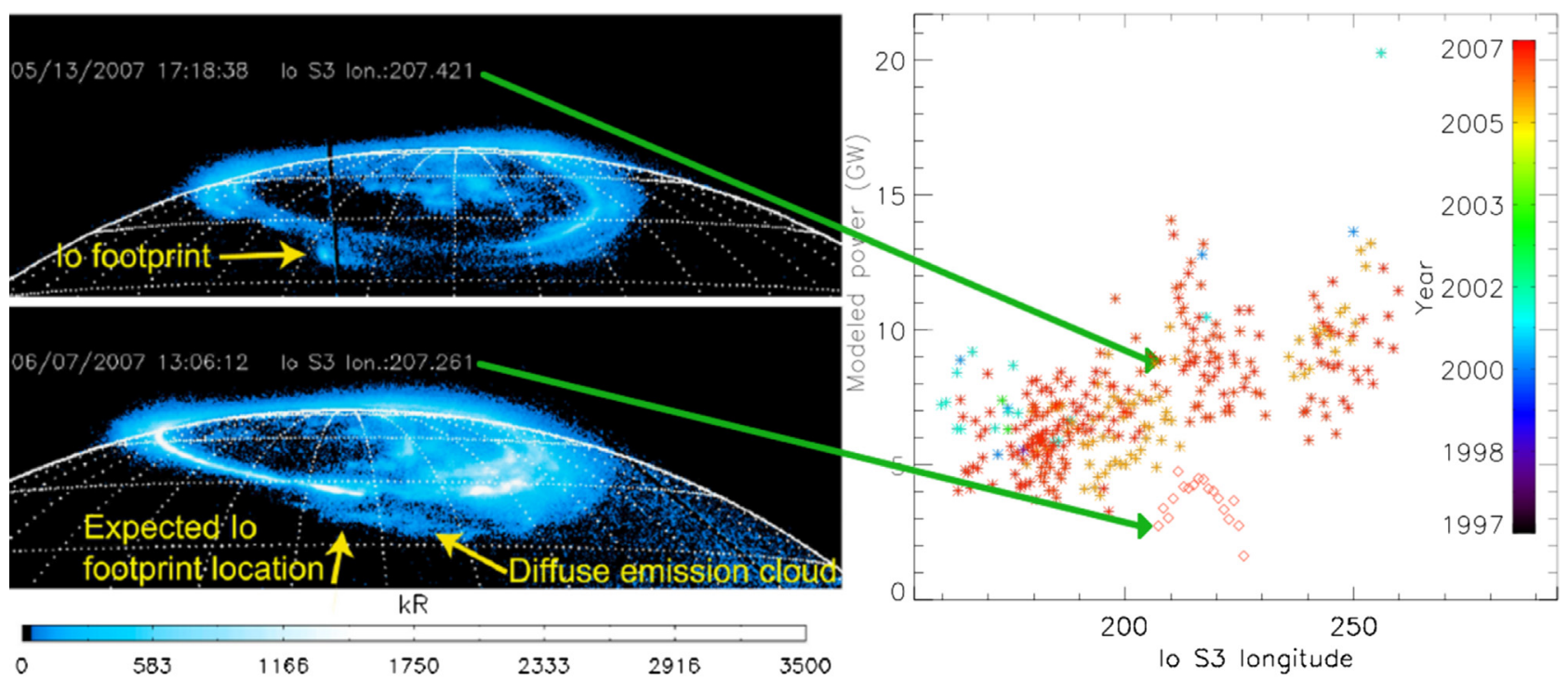

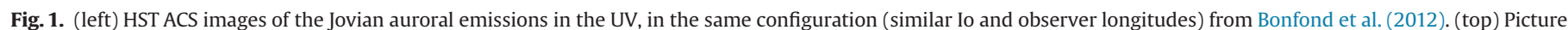

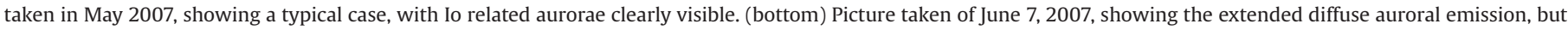

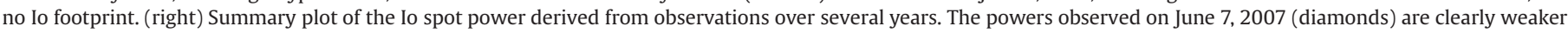
than the usual footprint powers at the same Io longitude.

Hubble Space Telescope can observe it as long as it is not behind the limb of the planet (Fig. 1). However, there is a unique occasion on which the Io footprint did not appear on an observation, although its theoretical location was visible. On June 7, 2007, HST observed the Jovian aurorae and should have observed the Io footprint on the northern hemisphere of Jupiter, but did not observe any auroral spot that could be related to Io (Bonfond et al., 2012, Figure 1b). A careful study of the observation condition showed that at this single time, the Io footprint brightness was at least three times dimmer than its usual value for a similar configuration (Bonfond et al., 2012), which is well below the typical variation of the footprint brightness (Bonfond et al., in press, Figure 1c).

The lack of detection of the Io footprint followed an unusual auroral event, which took the form of a wide $(15,000$ by $6000 \mathrm{~km}$ on Jupiter) patch of emissions extending in latitude from the main oval down to the Io footpath. Emissions located equatorward of the main oval but distinct from the footprint have been associated with two different mechanisms: injection events which create patchy auroral features (Mauk et al., 2002), or a Pitch Angle Diffusion which creates diffuse auroral arcs (see Radioti et al., 2009). The auroral feature observed during the non-observation of Io appears like the remnant of a patch that was better defined the day before, suggesting that this auroral event was most probably caused by a large injection event of the Io torus, with flux tubes carrying a dense and cold plasma from the torus moving outward to the magnetosphere and being quickly replaced by flux tubes filled by a warmer and less dense plasma (see discussion in Bonfond et al., 2012). The remnant auroral emission indicates that even though the injection event occurred several hours before the Io footprint disappearance, accelerated electrons were still present in this longitude sector.

These so-called injection events are common in the Jovian inner magnetosphere (Mauk et al., 1999). Close to Io's orbit, their presence is deduced from in situ observations which show flux tubes depleted in plasma (Kivelson et al., 1997; Thorne et al., 1997; Russell et al., 2005) of small extent (a few hundreds to about one thousand kilometers). Measurements of the electron energy spectra in the inner plasma sheet (Frank and Paterson, 1999) suggest that the electron energy spectrum inside of these empty flux tubes may be similar to that in the Io flux tube - i.e. kappa-like distribution with a characteristic energy of a few hundreds of $\mathrm{eV}$ - and would hence be able to generate auroral emissions (Hess et al., 2011b). The event of June 7, 2007 is unique by the fact that an auroral event with such a large extent was never observed that deep in the Jovian inner magnetosphere before (i.e. down to Io's orbit).

Since the observation of the Io footprint disappearance, the question of how a feature as stable as the footprint of Io could experience such a dimming has been puzzling. The Io interaction with Jupiter is relatively well-known, with well-tested models of the power generation (Neubauer, 1980; Saur, 2004), of the power transfer (Hess et al., 2010) and of the auroral emission (Gustin et al., 2004). Hence, the parameters involved in the determination of the footprint brightness are known as well. However, only a few parameters can sensibly vary, and some may have to vary by orders of magnitude to decrease the brightness of the footprint by a factor of three. In the following, we briefly summarize the model of the power generation and transfer between Io and Jupiter, as the details of the models have been published and discussed in several papers already (Section 2). Then, we select a limited set of relevant parameters, whose variation may be related to an injection event, and which appear to be able to modulate the footprint brightness according to the models (Section 3): $\rho_{\text {tor }}$ the density at the center of the torus; $H_{t o r}$ the scale-height of the torus; $\rho_{h l}$ the magnetospheric plasma density at high latitude; and $H_{\text {iono }}$ the scale-height of the Jovian ionosphere. These parameters are illustrated in Fig. 2, which shows the electron density profile used in Hess et al. (in press) for Io at a longitude of $210^{\circ}$, the longitude near which the Io footprint dimming occurred. The results indicate that the June 7, 2007 observation is most probably due to the increase of the electron content of the Io flux tube at high latitude. This can be caused by an increase of the supra-thermal electron population in the plasma torus $\left(\rho_{h l}\right)$ and to a lesser extent by the heating and vertical expansion of the Jovian upper ionosphere (increase of $H_{\text {iono }}$ ) caused by an injection event in the inner magnetosphere.

\section{Model of the current system}

An electric field exists across Io due to its motion relative to the magnetic field $\left(E_{\mathrm{Io}}=-V_{\mathrm{Io}} \times B\right)$. As Io possesses a conductive 


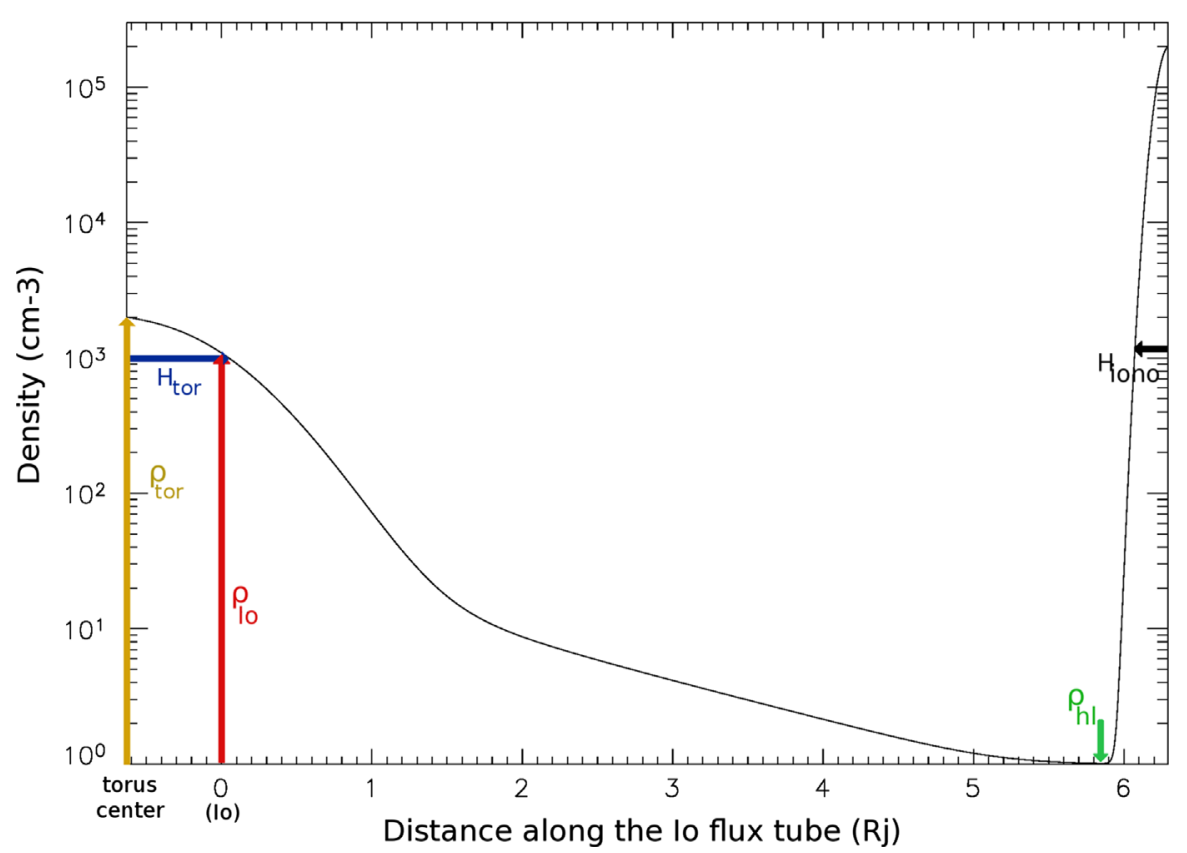

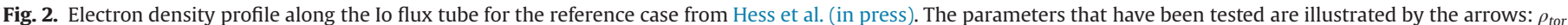

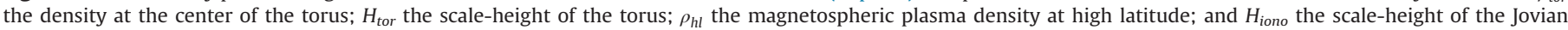
ionosphere. The arrow standing for $H_{\text {iono }}$ is not to scale, as it would then be too small to be visible in the figure. The density at Io, $\rho_{\text {Io }}$, is also indicated by an arrow.

ionosphere, this electric field generates a current across Io, which in turn creates a perturbation of the magnetic field (Alfvén wave packet) close to Io. As the travel time of an Alfvén wave to Jupiter is several times longer than the time it takes for a magnetic field line to pass Io (Delamere et al., 2003), the current is not in steady-state, but mostly transient (Neubauer, 1980; Goertz, 1983; Hess et al., 2011c). As a consequence, remote conductances - e.g. the Jovian ionosphere conductance - do not impact the current generated at Io, and the current can be deduced from the electric field across Io and the height-integrated Alfvén conductance $\Sigma_{A}$ (Neubauer, 1980; Goertz, 1983; Saur, 2004). The current density per hemisphere is

$J=4 E_{\mathrm{Io}} R_{\mathrm{Io}} \Sigma_{A} \simeq 4 V_{\mathrm{Io}} R_{\mathrm{Io}} \sqrt{\frac{\rho_{\mathrm{Io}}}{\mu_{0}}}$

Note that the current expression only depends on local values, identified by the Io subscript. The density at Io, $\rho_{\text {Io }}$, can be obtained from that at the torus center, $\rho_{\text {tor }}$, from the torus scaleheight, $H_{t o r}$, and from the distance between Io and the torus center. For the event considered here, Io is about $0.7 R_{J}$ above the torus center. The current is mostly carried by an Alfvén wave packet propagating at the Alfvén velocity, $V_{a}$, toward Jupiter's ionosphere, where the current system finally closes. From the expressions of the current and electric field across Io, one can derive the power $(P w)$ generated per hemisphere at Io by the interaction of the satellite with the magnetosphere (Saur, 2004)

$P_{w} \simeq 8 V_{\mathrm{Io}}^{2} R_{\mathrm{Io}}^{2} B_{\mathrm{Io}} \sqrt{\frac{\rho_{\mathrm{Io}}}{\mu_{0}}}$

That is not the power reaching the Jovian magnetosphere however, as Alfvén waves are partially reflected on the torus borders due to strong gradients of the plasma density $\rho$ affecting the Alfvén velocity $V_{a}$ (Gurnett and Goertz, 1981)

$V_{a}=\sqrt{\frac{B^{2}}{\mu_{0} \rho}}$

Upon reflection, a fraction of the wave and thus a part of the current are reflected back in the torus, so that the current at
Jupiter is smaller than that generated at Io. The transmission of a wave through a refraction index gradient is generally computed assuming that the ratio between the wavelength and the gradient characteristic scale is either small or large (WKB or discontinuity approximations). The wavelength spectrum of the Alfvén waves generated by the interaction - which peaks around the Io scale covers an intermediate range (Wright, 1987). In terms of spectral power carried by the Alfvén waves $\left(\mathcal{P}_{w}\right)$, the approximated reflection coefficient is given by Hess et al. (2010, in press)

$\frac{d \mathcal{P}_{w}}{d s}(s, \mathbf{k})=\frac{-\mathcal{P}_{w}(s, \mathbf{k})}{\lambda_{\|}}\left(\int_{s-\lambda_{\|} / 2}^{s+\lambda_{\|} / 2} \frac{\nabla_{s} \ln \left(c / V_{\phi, a}(\mathbf{k})\right)}{2} d s\right)^{2}$

where $s$ is the curvilinear distance along the magnetic field lines. Eq. (4) is explicitly dependent on the parallel wavelength $\left(\lambda_{\|}\right)$. The dependence on the perpendicular wavelength occurs through the phase velocity $V_{\phi, a}$ but can be neglected as $V_{\phi, a} \simeq V_{a}$ over most of the spectrum (Hess et al., 2010). This equation implies that short wavelengths are slightly reflected whereas long wavelengths are strongly reflected.

It is assumed that a given flux tube acts as a wave-guide for the Alfvén waves. As the magnetic field lines converge toward the planet, the cross-section of the flux tube decreases, implying an increase of the perpendicular wavevector. Close to Jupiter, the convergence of the magnetic field lines forces the Alfvén perpendicular wavelength toward small values, close to that of the electron inertial length, so that a parallel electric field appears due to kinetic effects and accelerates electrons (Lysak and Song, 2003; Swift, 2007; Hess et al., 2010). The parallel electric field generated by an inertial Alfvén wave can be approximated by Lysak and Song (2003)

$\delta E_{\|} \simeq \omega_{a} \sqrt{\mu_{a c c}} k_{\perp ; \mathrm{Io}} \lambda_{e}^{2} \delta B$

where $\omega_{a}$ is the Alfvén frequency, $\lambda_{e}$ is the electron inertial length, $k_{\perp}$ the Alfvén perpendicular wavevector, $\delta B$ the magnetic field perturbation associated with the wave and $\mu_{a c c}$ is the mirror ratio, i.e. the magnetic flux ratio, between the acceleration region and the equatorial plane. The profile along the magnetic field lines of the amplitude of the parallel electric field associated with the 
Alfvén wave presents a narrow peak just above the Jovian ionosphere (i.e. at an altitude of $\sim 0.5 R_{J}$ ), which is likely the cause of the electron acceleration (Hess et al., 2010). The localization of the electric field generates impulsive accelerations of the electrons (Swift, 2007; Hess et al., 2007), both toward Jupiter and in the antiplanetward direction, which is consistent with the observed characteristics of the footprint emissions (Hess et al., 2007; Bonfond, 2010), and the observations of electron beams near Io (Williams and Thorne, 2003, and references therein).

The power transferred to the electrons by an Alfvén wave packet can be estimated by assuming that, for each Alfvén wavelength, the electrons are accelerated by the parallel electric field associated to the Alfvén wave during a half-period of the wave. Hess et al. (2010) showed that in this case, an Alfvén wave carrying a power $\mathcal{P}_{w}\left(s_{a c c}, \mathbf{k}\right)$ transfers to the electrons a power $\mathcal{P}_{e}(\mathbf{k})$ given by

$\frac{\mathcal{P}_{e}(\mathbf{k})}{\mathcal{P}_{w}\left(S_{a c c}, \mathbf{k}\right)}=\min \left(\frac{\pi^{2}}{8} \frac{V_{t h} \mu_{a c c} k_{\perp ; \mathrm{Io}}^{2} \lambda_{e}^{2}}{V_{\phi ; a}(\mathbf{k})} ; 1\right)$

where the electrons to be accelerated have a thermal velocity $V_{t h}$ and an inertial length $\lambda_{e}$.

One can separate the propagation effects (Eq. (4), which mainly depends on $k$ ) and the electron acceleration (Eq. (6), which depends on $k_{\perp}$ ) to link the total power transmitted to the electrons $\left(P_{e}=\int \mathcal{P}_{e}(k) d k\right)$ to the total power generated at Io $\left(P_{w}=\int \mathcal{P}_{w}(0, k) d k\right)$ given by Eq. (2). To do so however, it is necessary to know the distribution of the Alfvén wavevectors. It has been shown by Hess et al. (2010) that this distribution should be a power law, corresponding to the turbulent filamentation of a Gaussian wavepacket peaking around the Io scale. The spectra indices range between $-5 / 3$ and -2 , and were chosen to be -2 for perpendicular wavevectors and $-5 / 3$ for parallel one by Hess et al. (in press, see discussion therein). The latter authors studied the evolution of the IFP brightness with Io's longitude. We use the same parameters as those authors, including the Alfvén wavevector distribution, because these parameters allowed Hess et al. to reproduce the correct brightness of the Io spot, and so that these two papers give a complete overview of the dependency of the satellite footprint brightness on the plasma parameters. The Jovian magnetic field model we use is the VIPAL model (Hess et al., 2011a).

\section{How could the Io footprint disappear?}

\subsection{Parameter study: parameter selection}

The disappearance of the Io footprint, or more precisely the dimming of it brightness by at least a factor of three, followed an auroral episode which is assumed to be associated with an injection event, i.e. with the outward ejection of flux tubes containing a dense and cold plasma, replaced by flux tubes containing a tenuous and warm plasma. Thus, the primary parameters to be investigated are the density and temperature profiles of the electrons. More precisely, following the injection event, one could expect (1) a decrease of the torus density $\rho_{\text {tor }}$ (dense plasma replaced by tenuous plasma), (2) an increase of the torus electron temperature (cold plasma replaced by warm plasma) and thus of the characteristic scale of the Alfvén velocity gradient (through the scale-height of the torus $H_{t o r}$ ), (3) an increase of the magnetospheric plasma density out of the torus $\rho_{h l}$ (a warmer plasma has a larger scale-height) and (4) a larger scale-height of the Jupiter ionosphere $H_{\text {iono }}$ (an intense auroral event can heat the ionosphere), and thus a higher altitude of the acceleration region.
The density of the Io plasma torus, $\rho_{\text {tor }}$, has been modeled by Bagenal (1994) and, Moncuquet et al. (2002) from spacecraft observations. These study shows that the equatorial electron density profile peaks close to Io's orbit at about $2000 \mathrm{~cm}^{-3}$. Moving outward, the electron density decreases slowly to about $700-800 \mathrm{~cm}^{-3}$ at a distance of $7.5 R_{J}$ from Jupiter's center. Between 7.5 and $8 R_{\mathrm{J}}$, the electron density experiences an abrupt decrease down to $100 \mathrm{~cm}^{-3}$. The density at Io cannot be lower than the density at larger distances, and it is unlikely that all the plasma occupying the volume between Io's orbit and $8 R_{J}$ get expelled at once, hence the torus density cannot be much lower than $1000 \mathrm{~cm}^{-3}$. On the contrary, Io volcanic activity is not constant, and strong eruptions can lead to torus densities larger than the average one by at least a factor of two (Bagenal et al., 1997), so that torus densities up 5000-6000 $\mathrm{cm}^{-3}$ may be possible (see Galileo measurements in Moncuquet et al., 2002).

The scale-height of the torus, $H_{t o r}$, is a consequence of the inertial confinement of the torus due to centrifugal forces. These forces are constant, so that the torus scale-height can only be modulated by the torus plasma temperature. The core electron temperature in the torus is about $5 \mathrm{eV}$, and is observed to vary from $-30 \%$ to $+40 \%$ (Steffl et al., 2004). Hence, the torus scale height may vary in the same proportions. The scale-height variation is observed to be anticorrelated to the torus density variation, a $10 \%$ higher temperature meaning a $\sim 10 \%$ lower density (Steffl et al., 2004).

The electron density at high-latitude (i.e. at the position of the acceleration region) is less-constrained, being estimated as equal to the $\mathrm{H}^{+}$density in the torus, that is $\rho_{h l} \sim 1 \mathrm{~cm}^{-3}$. This density could however be highly variable. The ambipolar electric field confining the electrons inside the torus has been estimated by Su et al. (2003) to be about $60 \mathrm{~V}$. This is sufficient to confine the $5 \mathrm{eV}$ electron in the torus. But this ambipolar field is weak enough so that warm electron populations carried by inward moving flux tubes or accelerated by auroral processes may ignore it, so that these populations are not confined in the torus at all and fully participate to the density at high latitude. Hence, this density may easily vary by an order of magnitude. However, as even inward moving flux tubes filled with warm plasma do not contain much more than one hundred electrons per $\mathrm{cm}^{3}$ (Russell et al., 2005), it is unlikely that the high latitude density exceeds a few tens of electrons per $\mathrm{cm}^{3}$.

There has been several estimates of the Jovian ionosphere scale-height, $H_{\text {iono, }}$ involving several kinds of models and methods of observations. These estimates range from $500 \mathrm{~km}$ up to $2000 \mathrm{~km}$ (Eshleman et al., 1979; Grodent et al., 2001; Lystrup et al., 2008) and recent measurements of the modulations of the Io-related radio emissions, which are related to the eigenfrequencies of the Alfvén ionospheric resonator (Su et al., 2006), suggested that the ionosphere inside the Io footprint has a scale height of $\sim 1600 \mathrm{~km}$ (Koshida et al., 2010), but it has been noted that after an auroral event, the scale-height can sensibly increase (Grodent et al., 2001; Su et al., 2003; Lystrup et al., 2008) so that this value may be larger than the characteristic one. In the reference model of Hess et al. (in press) the scale-height is slightly larger than $1000 \mathrm{~km}$, hence being consistent with the measurements and models. The ionosphere scale height may then vary by a factor of two at most.

\subsection{Parameter study: results}

Fig. 3 shows the dependency of the transmission of the power from Io to the acceleration region on the above parameters. The crosses show the Hess et al. (in press) values which gave IFP brightnesses consistent with that typically observed. The June 7 , 2007 case corresponds to brightnesses at least three times 

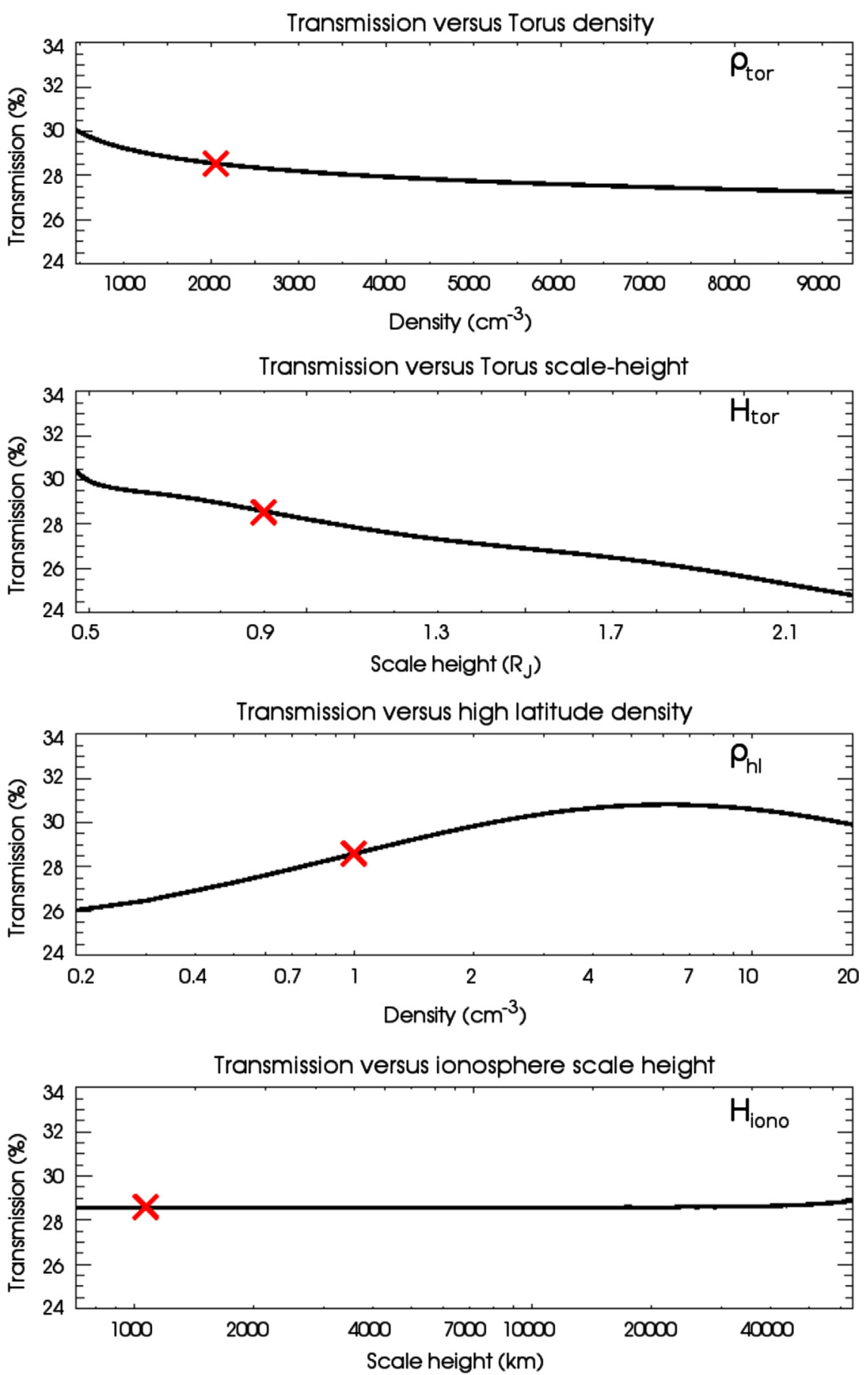

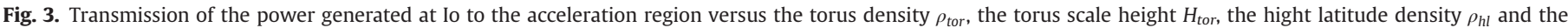

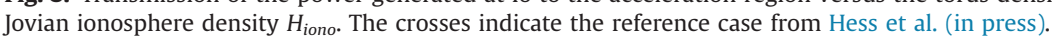

dimmer. As one can expect, the power transmission depends weakly on the ionospheric scale-height, $H_{\text {iono }}$, and on the density at high latitude, $\rho_{h l}$, except when the latter becomes very low $\left(<1 \mathrm{~cm}^{-3}\right)$ in which case the gradient of refraction index on the torus border becomes sensibly higher, preventing the Alfvén wave from escaping the torus. The dependency is also relatively low on the torus density, $\rho_{\text {tor }}$, because two effects are competing: a larger density means a larger refraction index gradient on the torus border and less transmission, but it also means a smaller Alfvén velocity and hence smaller parallel wavelengths and more transmission.

The torus scale-height $H_{\text {tor }}$ sensibly modifies the power transmission, not by changing the characteristic scale of the refraction index gradient on the torus borders (otherwise the transmission would be larger for long scales), but because of the position of Io relative to the torus. When Io is at a longitude of $210^{\circ}$, the satellite is also located about $0.7 R_{J}$ above the torus center. This distance also corresponds approximately to the scale-height of the torus. Hence, when the scale-height decreases Io passes above the region of strong gradient (so there are little reflections), but when the scale-height increases Io passes below it and the transmission decreases.

Fig. 4 shows the dependency of the efficiency of the power transfer to the electrons for the same parameters. The torus parameters, $\rho_{\text {tor }}$ and $H_{\text {tor }}$, do not impact this efficiency, because the acceleration occurs far from the torus. On the contrary, the 

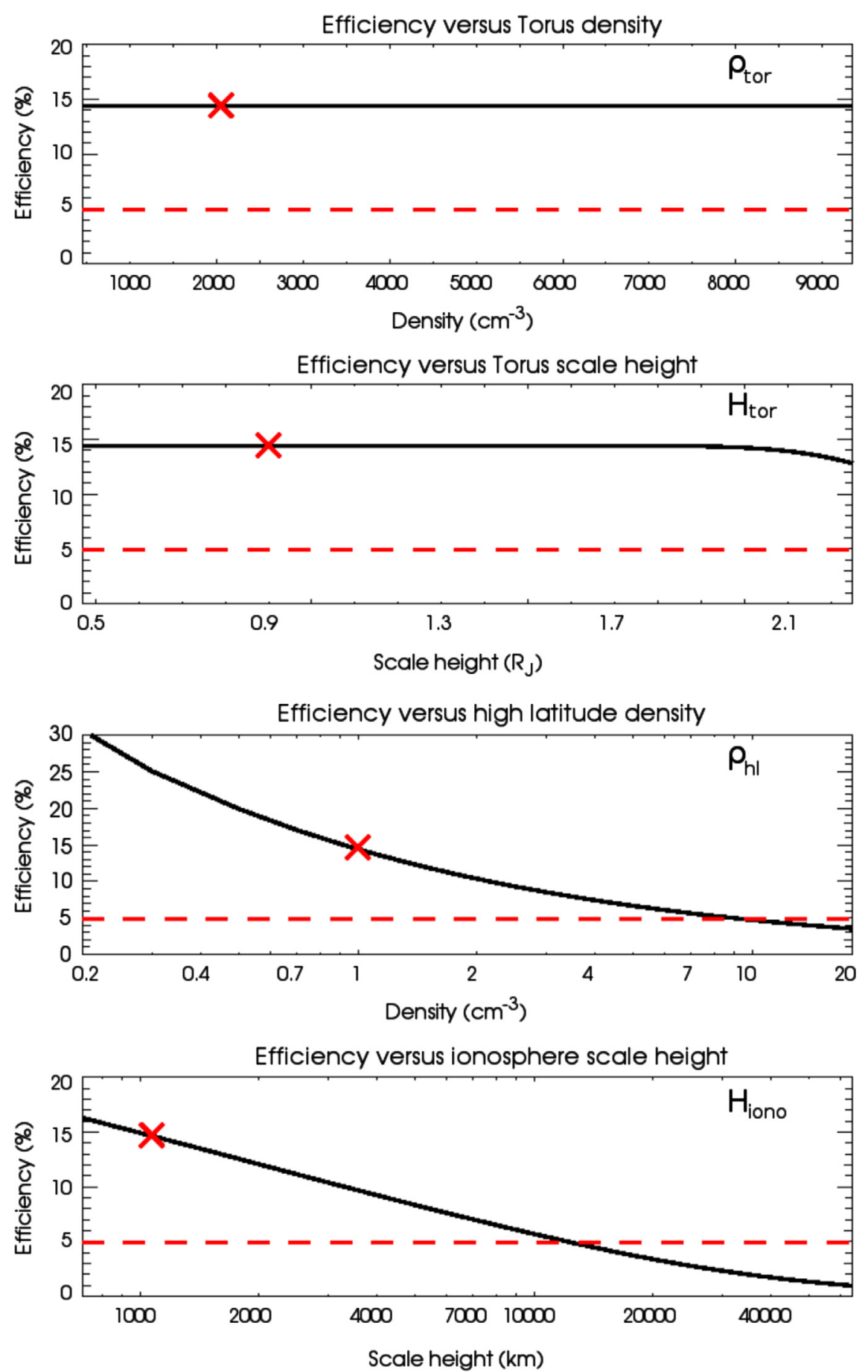

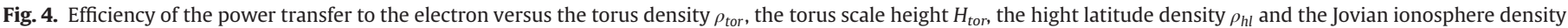
$H_{\text {iono. }}$ The crosses indicate the reference case from Hess et al. (in press) and the dashed lines indicate the third of the reference case brightness.

magnetospheric plasma density at high latitude, $\rho_{h l}$, and the Jovian ionosphere scale-height, $H_{\text {iono }}$, change drastically the efficiency of the electron acceleration (Eq. (6)), because they change the electron inertial length $\lambda_{e}$ and the altitude of the acceleration (and hence the mirror ratio $\mu_{a c c}$ ), respectively.

Finally, Fig. 5 shows the power transferred to the electrons, versus the same parameters. These results include both the variation of the power transmission and transfer to the electrons, as well as the modulation of the power generated at Io. In this latter figure, the variation amplitudes are larger than in previous figures for all of the parameters. The reason for it is obvious in the case of the torus density, $\rho_{\text {tor }}$, since the power generated at Io directly depends on this parameter $\left(\rho_{\text {Io }} \propto \rho_{\text {tor }}\right.$, Eq. (2)). In the case of the torus scale-height, $H_{\text {tor }}$, the power precipitated increases with the scale-height because at a longitude of $210^{\circ}$ Io is located at the northern border of the torus, so that when the torus scaleheight $\left(H_{t o r}\right)$ changes the density around Io $\left(\rho_{\mathrm{Io}}\right)$ changes too, modulating the power generated (Eq. (2)). The variation of the power precipitated versus the high latitude density $\rho_{h l}$ is mostly due to the efficiency of the power transfer to the electrons. Its variation versus the Jovian ionosphere scale-height $H_{\text {iono }}$ resembles that in Fig. 4 but with a larger amplitude. This is due to the 

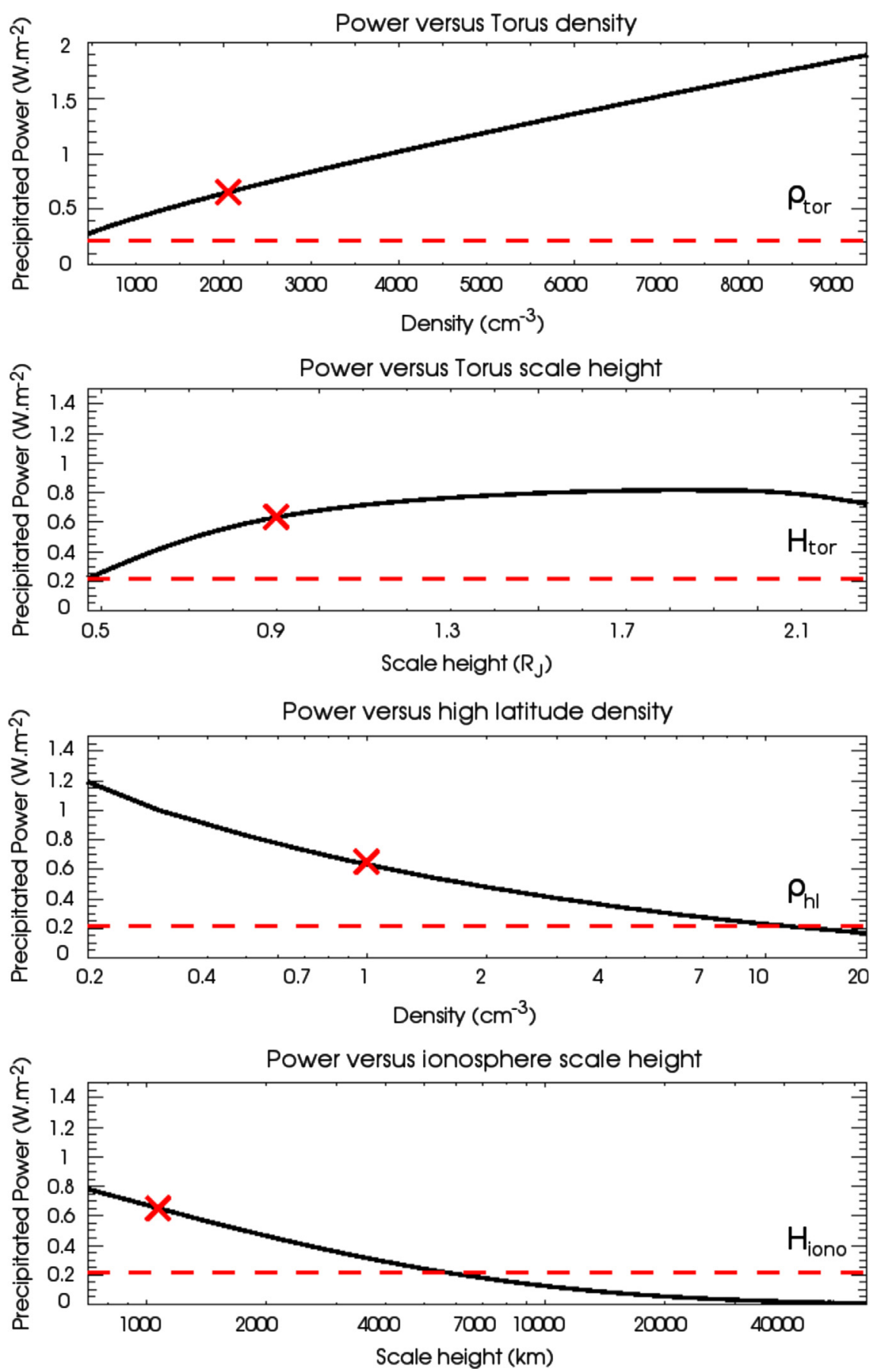

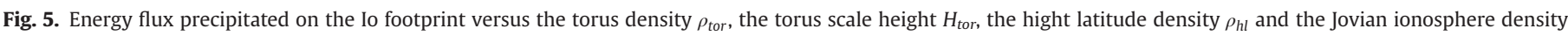
$H_{\text {iono. }}$ The crosses indicate the reference case from Hess et al. (in press) and the dashed lines indicate the third of the reference case brightness.

magnetic mirroring effect which reflects a larger number of particles when the acceleration is at a larger altitude (see discussion in Hess et al., in press).

\subsection{Discussion}

The above results show that the variation of all of the tested parameters could ultimately lead to a decrease of the Io footprint brightness by a factor of three. However, the variation amplitude needed is not the same for all of the parameters. A decrease of the torus density leads to a decrease of the footprint brightness, but for the brightness to decrease by a factor of three the density must decrease from $\rho_{\text {tor }} \sim 2000 \mathrm{~cm}^{-3}$ down to $\rho_{\text {tor }} \sim 300 \mathrm{~cm}^{-3}$.
The sense of the variation needed is consistent with an injection event, which releases plasma from the torus in the outer magnetosphere, but the amplitude of the release is quite large. At Jupiter, injection events correspond to interchanges between dense torus flux tubes and lighter flux tubes from larger distances. However, even the lighter flux tubes have densities of a few hundreds of $\mathrm{cm}^{-3}$. For the density at Io to be so low, all of the plasma from the torus should be replaced by empty flux tube over a large radial (6$8 R_{J}$ ) and longitudinal extent (several tens of degrees according to UV observations), whereas interchanged flux tubes usually cover less than $1 \%$ of the flux tubes (Russell et al., 2005). Even if we imagine that the event preceding the Io footprint disappearance corresponded to an exchange of flux tubes ten time larger than 
usual, i.e. if the interchanged flux tubes cover $\sim 10 \%$ of the torus, the averaged density in the torus would decrease by about $10 \%$ of the torus density, i.e. a couple hundreds of $\mathrm{cm}^{-3}$ at most. This would decrease a bit the brightness of the Io footprint, but by less than $10 \%$.

In order for the footprint brightness to decrease, the torus scale-height $H_{\text {tor }}$ must decrease, which corresponds to a cooling of the torus plasma. The temperature should decrease by a factor of two, which is far lower than what is usually seen (Steffl et al., 2004). Even though such a cooling occurred, the cooling of the torus usually involves an increase of the torus density $\rho_{\text {tor }}$ (Brown, 1995; Steffl et al., 2004) which would then increase the footprint brightness. For those reasons, a cooling of the plasma torus is not favored by our results.

The heating of the plasma during interchange events modeled by Hess et al. (2011b) is partly due to the acceleration of electrons at high latitude in the interchanged flux tube, in a way similar to that happening in the Io flux tube. The authors explained the temperature variations with the system III longitude observed in the plasma torus (Steffl et al., 2004) using the same model than in the present study. In particular, the authors showed that the warm electron production and life expectancy were maximum near a longitude of $270^{\circ}$, and is still higher than average at $210^{\circ}$. Hence, it is possible that the warm electron content of the flux tube, which corresponds to the density at high latitude $\rho_{h l}$, be much higher than normal for a large injection event such as the one observed before the Io footprint disappearance.

A dimming of the footprint brightness by a factor of three can be obtained if the warm electron content of the Io flux tube rises from $\rho_{h l} \sim 1 \mathrm{~cm}^{-3}$ to $\rho_{h l} \sim 9 \mathrm{~cm}^{-3}$. This may appear as a large factor, but the production of a few warm electrons per $\mathrm{cm}^{3}$ is easy, as discussed in Section 3.1 and flux tubes filled with warm electrons have been seen close to Io by Galileo (Frank and Paterson, 1999).

The injection event generated an auroral activity observed in the UV (Bonfond et al., 2012), meaning that a large amount of energy was deposited in the Jovian ionosphere, possibly heating it (Grodent et al., 2001). In that case, the scale-height of the ionosphere, which is $H_{\text {iono }} \simeq 1000 \mathrm{~km}$ in the reference model of Hess et al. (in press), can increase (Grodent et al., 2001; Su et al., 2003; Lystrup et al., 2008). The largest estimates of the ionosphere scale height never exceed $H_{\text {iono }} \sim 2000 \mathrm{~km}$ (Eshleman et al., 1979; Grodent et al., 2001; Lystrup et al., 2008). According to our calculations, an increase of the Jovian ionosphere scale-height to $2000 \mathrm{~km}$ would mean a decrease of the footprint brightness by about $30 \%$.

\section{Conclusion}

We used a well-tested model of the power transfer between Io and its auroral footprint on Jupiter to test which parameters could lead to an abrupt dimming of the footprint brightness. Such an event, although extremely rare, occurred on June 7, 2007, following a large injection event which was observed in the UV (Bonfond et al., 2012). It is assumed that the injection event caused the Io footprint disappearance by modifying the plasma parameter in the torus, or along the Io flux tube. After we varied several parameters (the density at the center of the torus; the scale-height of the torus; the magnetospheric plasma density at high latitude; and the scale-height of the Jovian ionosphere), our conclusions point toward an increase of the warm electron content of the flux tube and possibly an increase of the Jovian ionosphere scale-height and a smaller effect of the torus density decrease. Those parameters which can dim the footprint brightness by a factor of three by varying with an amplitude consistent with our understanding of the magnetosphere dynamics: if such a dimming was to be caused by a change in the torus only, the amplitude of the change would be such that it would require the torus to almost completely disappear. Moreover, both the high latitude density and ionospheric scale-height increases could be caused by a large injection event causing auroral emissions in the UV. However, a cooling of the torus is not excluded by our computations, but is not favored because it would be associated with an increase of the density which enhances the footprint brightness.

\section{References}

Bagenal, F., 1994. Empirical model of the Io plasma torus: Voyager measurements. Journal of Geophysical Research 99, 11043-11062.

Bagenal, F., 1997. Ionization source near Io from Galileo wake data. Geophysical Research Letters 24, 2111.

Bagenal, F., Crary, F.J., Stewart, A.I.F., Schneider, N.M., Gurnett, D.A., Kurth, W.S., Frank, L.A., Paterson, W.R., 1997. Galileo measurements of plasma density in the Io torus. Geophysical Research Letters 24, pp. 2119-+.

Bonfond, B., 2010. The 3-D extent of the Io UV footprint on Jupiter. Journal of Geophysical Research 115, pp. 9217-+

Bonfond, B., Gérard, J.C., Grodent, D., Saur, J., 2007. Ultraviolet Io footprint short timescale dynamics. Geophysical Research Letter 34, 6201.

Bonfond, B., Grodent, D., Gérard, J.C., Radioti, A., Dols, V., Delamere, P.A., Clarke, J.T., 2009. The Io UV footprint: location, inter-spot distances and tail vertical extent. Journal of Geophysical Research 114 7224-+.

Bonfond, B., Grodent, D., Gérard, J.C., Stallard, T., Clarke, J.T., Yoneda, M., Radioti, A. Gustin, J., 2012. Auroral evidence of Io's control over the magnetosphere of Jupiter. Geophysical Research Letters 39, 1105.

Bonfond, B., Hess, S.L.G., Grodent, D., Gérard, J.C., Radioti, A., Chantry, V., Saur, J., Jacobsen, S., Clarke, J.T. Evolution of the IFP brightness I: observations. Planetary and Space Sciences, http://dx.doi.org/10.1016/j.pss.2013.05.023, in press.

Brown, M.E., 1995. Periodicities in the Io plasma torus. Journal of Geophysical Research 696, 21683-21696.

Clarke, J.T., Ballester, G.E., Trauger, J., Evans, R., Connerney, J.E.P., Stapelfeldt, K., Crisp, D., Feldman, P.D., Burrows, C.J., Casertano, S., Gallagher III, J.S., Griffiths, R. E., Hester, J.J., Hoessel, J.G., Holtzman, J.A., Krist, J.E., Meadows, V., Mould, J.R., Scowen, P.A., Watson, A.M., Westphal, J.A., 1996. Far-ultraviolet imaging of Jupiter's Aurora and the lo "Footprint". Science 274, 404-409.

Connerney, J.E.P., Baron, R., Satoh, T., Owen, T., 1993. Images of excited $\mathrm{H}_{3}^{+}$at the foot of the Io flux tube in Jupiter's atmosphere. Science 262, 1035-1038.

Delamere, P.A., Bagenal, F., Ergun, R., Su, Y.J., 2003. Momentum transfer between the Io plasma wake and Jupiter's ionosphere. Journal of Geophysical Research 108, pp. 11-1.

Eshleman, V.R., Tyler, G.L., Wood, G.E., Lindal, G.F., Anderson, J.D., Levy, G.S., Croft, T, A., 1979. Radio science with Voyager 1 at Jupiter - Preliminary profiles of the atmosphere and ionosphere. Science 204, 976-978.

Frank, L.A., Paterson, W.R., 1999. Intense electron beams observed at Io with the Galileo spacecraft. Journal of Geophysical Research 104, pp. 28657-+.

Goertz, C.K., 1983. The Io-control of Jupiter's decametric radiation - The Alfven wave model. Advances in Space Research 3, 59-70.

Goldreich, P., Lynden-Bell, D., 1969. Io, a Jovian unipolar inductor. Astrophysics Journal 156, 59-78.

Grodent, D., Waite, J.H., Gérard, J.C., 2001. A self-consistent model of the Jovian auroral thermal structure. Journal of Geophysical Research 106, 12933-12952.

Gurnett, D.A., Goertz, C.K., 1981. Multiple Alfven wave reflections excited by Io Origin of the Jovian decametric arcs. Journal of Geophysical Research 86, 717-722.

Gustin, J., Gérard, J.C., Grodent, D., Cowley, S.W.H., Clarke, J.T., Gérard, A., 2004. Energy-flux relationship in the FUV Jovian aurora deduced from HST-STIS spectral observations. Journal of Geophysical Research (Space Physics) 109, 10205.

Hess, S., Mottez, F., Zarka, P., 2007. Jovian S-bursts generation by Alfvén waves. Journal of Geophysical Research 112, A11212.

Hess, S.L.G., Bonfond, B., Chantry, V., Gérard, J.C., Grodent, D., Jacobsen, S., Radioti, A. Evolution of the Io footprint brightness II: modeling. Planetary and Space Science, http://dx.doi.org/10.1016/j.pss.2013.08.005, in press.

Hess, S.L.G., Bonfond, B., Zarka, P., Grodent, D., 2011a. Model of the Jovian magnetic field topology constrained by the Io auroral emissions. Journal of Geophysical Research 116, A05217.

Hess, S.L.G., Delamere, P., Dols, V., Bonfond, B., Swift, D., 2010. Power transmission and particle acceleration along the Io flux tube. Journal of Geophysical Research 115, pp. 6205-+.

Hess, S.L.G., Delamere, P.A., Bagenal, F., Schneider, N., Steffl, A.J., 2011b. Longitudinal modulation of hot electrons in the Io plasma torus. Journal of Geophysical Research 116, 11215.

Hess, S.L.G., Delamere, P.A., Dols, V., Ray, L.C., 2011c. Comparative study of the power transferred from satellite-magnetosphere interactions to auroral emissions. Journal of Geophysical Research 116, A01202. 
Kivelson, M.G., Khurana, K.K., Russell, C.T., Walker, R.J., 1997. Intermittent shortduration magnetic field anomalies in the Io torus: evidence for plasma interchange?. Geophysical Research Letter 24, pp. 2127-+.

Koshida, T., Shibata, T.F., Tagushi, S., Misawa, H., 2010. Characteristics of Jovian ionospheric Alfvén resonator observed by using wave modulations of L-burst emissions. Journal of Geophysical Research 115, A12213.

Lysak, R.L., Song, Y., 2003. Kinetic theory of the Alfvén wave acceleration of auroral electrons. Journal of Geophysical Research 108 6-1.

Lystrup, M.B., Miller, S., Dello Russo, N., Vervack Jr., R.J., Stallard, T., 2008. First vertical ion density profile in Jupiter's auroral atmosphere: direct observations using the Keck II telescope. Astrophysics Journal 677, 790-797.

Mauk, B.H., Clarke, J.T., Grodent, D., Waite, J.H., Paranicas, C.P., Williams, D.J., 2002. Transient aurora on Jupiter from injections of magnetospheric electrons. Nature 415, 1003-1005.

Mauk, B.H., Williams, D.J., McEntire, R.W., Khurana, K.K., Roederer, J.G., 1999. Stormlike dynamics of Jupiter's inner and middle magnetosphere. Journal of Geophysical Research 104, 22759-22778.

Moncuquet, M., Bagenal, F., Meyer-Vernet, N., 2002. Latitudinal structure of outer Io plasma torus. Journal of Geophysical Research 107 24-1.

Neubauer, F.M., 1980. Nonlinear standing Alfven wave current system at Io Theory. Journal of Geophysical Research 85, 1171-1178.

Prangé, R., Rego, D., Southwood, D., Zarka, P., Miller, S., Ip, W., 1996. Rapid energy dissipation and variability of the Io-Jupiter electrodynamic circuit. Nature 379, 323-325.

Radioti, A., Tomás, A.T., Grodent, D., Gérard, J.C., Gustin, J., Bonford, B., Krupp, N., Woch, J., Menietti, J.D., 2009. Equatorward diffuse auroral emissions at Jupiter: simultaneous HST and Galileo observations. Geophysical Review Letter 36, 7101.
Russell, C.T., Kivelson, M.G., Khurana, K.K., 2005. Statistics of depleted flux tubes in the Jovian magnetosphere. Planetary and Space Science 53, 937-943.

Saur, J., 2004. A model of Io's local electric field for a combined Alfvénic and unipolar inductor far-field coupling. Journal of Geophysical Research 109, pp. $1210-+$.

Saur, J., Strobel, D.F., Neubauer, F.M., Summers, M.E., 2003. The ion mass loading rate at Io. ICARUS 163, 456-468.

Schneider, N.M., Trauger, J.T., 1995. The structure of the Io torus. Astrophysics Journal 450, pp. 450-+.

Steffl, A.J., Stewart, A.I.F., Bagenal, F., 2004. Cassini UVIS observations of the Io plasma torus. I. Initial results. Icarus 172, 78-90.

Su, Y.J., Ergun, R.E., Bagenal, F., Delamere, P.A., 2003. Io-related Jovian auroral arcs: modeling parallel electric fields. Journal of Geophysical Research 108, pp. 15-1.

Su, Y.J., Jones, S.T., Ergun, R.E., Bagenal, F., Parker, S.E., Delamere, P.A., Lysak, R.L. 2006. Io-Jupiter interaction: Alfvén wave propagation and ionospheric Alfvén resonator. Journal of Geophysical Research 111, pp. 6211-+.

Swift, D.W., 2007. Simulation of auroral electron acceleration by inertial Alfvén waves. Journal of Geophysical Research 112 12207-+.

Thomas, N., Bagenal, F., Hill, T.W., Wilson, J.K., 2004. The Io neutral clouds and plasma torus. In: Jupiter: The Planet, Satellites and Magnetosphere, pp. 561591.

Thorne, R.M., Armstrong, T.P., Stone, S., Williams, D.J., McEntire, R.W., Bolton, S.J., Gurnett, D.A., Kivelson, M.G., 1997. Galileo evidence for rapid interchange transport in the Io torus. Geophysical Research Letter 24 2131-+.

Williams, D.J., Thorne, R.M., 2003. Energetic particles over Io's polar caps. Journal of Geophysical Research 108, pp. 1397-+.

Wright, A.N., 1987. The interaction of Io's Alfven waves with the Jovian magnetosphere. Journal of Geophysical Research 92, 9963-9970. 